\title{
Male-specific cAMP signaling in the hippocampus controls spatial memory deficits in a mouse model of autism and intellectual disability
}

\author{
Marta Zamarbide ${ }^{1,{ }^{\star}}$, Adele Mossa ${ }^{1,}$, Pablo Muñoz-Llancao ${ }^{1}$, Molly K. Wilkinson ${ }^{1}$, Heather L. \\ Pond $^{1}$, Adam W. Oaks ${ }^{1}$, and M. Chiara Manzini ${ }^{1}$ \\ ${ }^{1}$ Institute for Neuroscience and Department of Pharmacology and Physiology, The George \\ Washington University School of Medicine and Health Sciences, Washington, DC, USA
}

\section{Abstract}

Background.-The prevalence of neurodevelopmental disorders is biased towards males with male:female ratios of 2:1 in intellectual disability (ID) and 4:1 in autism spectrum disorder (ASD). However, the molecular mechanisms of such bias remain unknown. While characterizing a mouse model for loss of the signaling scaffold coiled-coil and C2 domain containing 1A (CC2D1A), which is mutated in ID and ASD, we identified biochemical and behavioral differences between males and females, and explored whether CC2D1A controls male-specific intracellular signaling.

Methods.-CC2D1A is known to regulate phosphodiesterase 4D (PDE4D) which regulates cAMP signaling. We tested for activation PDE4D and downstream signaling molecules in the hippocampus of Cc2d1a-deficient mice. We then performed behavioral studies in females to analyze learning and memory, and then targeted PDE4D activation with a PDE4D inhibitor to define how changes in cAMP levels affect behavior in males and females.

Results.-We found that in Cc2d1a-deficient males PDE4D is hyperactive leading to a reduction in CREB signaling, but this molecular deficit is not present in females. Cc2d1a-deficient males show a deficit in spatial memory which is not present in $C c 2 d 1 a$-deficient females. Restoring PDE4D activity using an inhibitor rescues cognitive deficits in males, but has no effect on females.

Conclusions.-Our findings show that CC2D1A regulates cAMP intracellular signaling in a malespecific manner in the hippocampus leading to male-specific cognitive deficits. We propose that male-specific signaling mechanisms are involved in establishing sex bias in neurodevelopmental disorders.

Corresponding author: M. Chiara Manzini, The George Washington University, 2300 Eye Street NW, Ross Hall 650, Washington, DC 20037, Phone: +1 202-994-5924, cmanzini@ gwu.edu.

These authors contributed equally

FINANCIAL DISCLOSURES

The authors report no biomedical financial interests or potential conflicts of interest.

Publisher's Disclaimer: This is a PDF file of an unedited manuscript that has been accepted for publication. As a service to our customers we are providing this early version of the manuscript. The manuscript will undergo copyediting, typesetting, and review of the resulting proof before it is published in its final citable form. Please note that during the production process errors may be discovered which could affect the content, and all legal disclaimers that apply to the journal pertain. 


\section{Keywords}

autism; intellectual disability; sex bias; intracellular signaling; learning and memory; cAMP

\section{INTRODUCTION}

Developmental disabilities are more prevalent in males with male:female ratios ranging between 1.5-2:1 in intellectual disability (ID) and 4-8:1 in autism spectrum disorders (ASD) $(1 ; 2)$. However, the molecular mechanisms underlying sex bias in neurodevelopmental disorders remain unknown. The male and female brain show developmental sex differences in normal subjects beyond sex-specific behaviors such as mating and aggression (3). Noninvasive imaging studies in humans have shown that male and female brains have different patterns of connection (4), and comparison of activation of brain areas involved in social cognition in children with ASD found deficits only in affected boys, and not in girls (5). Lesion studies testing learning and memory in rodents showed that certain brain regions result in different deficits in males and females $(6 ; 7)$. In addition, males and females have been shown to use different cellular and molecular strategies to encode information for the same behaviors during memory acquisition (8;9). Intracellular signaling differs in males and females, and is a candidate for differentially controlling behavioral outputs. Activity of cAMP response element binding protein (CREB) downstream of protein kinase A (PKA) and mitogen-activated protein (MAPK/ERK) kinases is differentially regulated by estrogen in the hippocampus of males and females throughout development $(10 ; 11)$.

Here, we introduce the signaling scaffold coiled-coil and $C 2$ domain containing $1 A$ $(\mathrm{CC} 2 \mathrm{D} 1 \mathrm{~A})$, as a regulator of sex-specific intracellular signaling in the male hippocampus controlling spatial memory acquisition. Biallelic mutations in $C C 2 D 1 A$ cause a spectrum of neurodevelopmental conditions in humans with fully penetrant ID and variably penetrant ASD, ADHD, seizures, and aggressive behavior (12-15). While global Cc2d1a knock-out pups die at birth due to respiratory deficits, we found that conditional removal of this gene in the cortex and hippocampus leads to cognitive and social deficits, hyperactivity, and anxiety in males (16). CC2D1A was first described as a signaling and transcriptional regulator (17; 18 ), and it has the structure of a scaffold with multiple protein binding domains. In vitro studies in mouse embryonic fibroblasts and hippocampal neurons from $C c 2 d 1$ a global KO mice showed that it binds to phosphodiesterase (PDE4D), an enzyme involved in cAMP degradation, and regulates PDE4D activity and subcellular distribution $(19 ; 20)$. When cultured neurons lacking $C c 2 d 1 a$ are treated with forskolin to increase cAMP levels and activate CREB, hyperactive PDE4D reduces the effect of this drug leading to reduced PKA and CREB activation (19). We wondered whether PDE4D and CREB function was perturbed in the Cc2d1a-deficient hippocampus and whether these changes caused the spatial cognitive deficits observed in the mice. During our studies, we found that only Cc2d1a-deficient males show disruptions in PDE4D signaling, which correlate with malespecific spatial memory deficits. We then used a PDE4D inhibitor to rescue these deficits confirming that restoring signaling directly affects behavior. Females do not show these molecular and behavioral impairments. Our results show that CC2D1A regulates cAMP 
signaling in a sex-specific manner in males, revealing a novel mechanism controlling malespecific intracellular signaling and behavior.

\section{METHODS AND MATERIALS}

\section{Animals}

All animal care and use was in accordance with institutional guidance and approved by the Institutional Animal Care and Use Committee of The George Washington University. The Cc2d1a conditional knock-out (cKO) mouse line was generated by crossing Cc2d1a-flx mice (16) with a CaMKIIa-cre mouse line driving Cre recombinase expression under the CaMKIIa promoter (Stock 005359, Jackson Laboratories) (21). Global Cc2d1a and Cc2d1b knock-out (KO) mice were obtained from the Knock Out Mouse Project and were previously described $(16 ; 22)$. All animals are fully backcrossed on a C57BL/6J background for at least 6 generations. Since the CaMKIIa-cre transgene can be occasionally activated in male germ cells leading to germline transmission (23), crosses were conducted between homozygous Cc2d1a-flx males and double heterozygous Cc2d1a-flx/CaMKIIa-cre females. For genotyping, polymerase chain reaction (PCR) amplifications were performed on $1 \mu \mathrm{L}$ of proteinase K (New England Biolabs, Ipswich MA) digested tail DNA samples. Primer sequences are available upon request.

\section{Behavioral tests}

A standardized battery of behavioral testing was used for cKO animals at 3-4 months of age. Behavioral tests were performed in the Manzini lab behavior analysis suite in the George Washington University Animal Research Facility following a $60 \mathrm{~min}$ period of acclimatization. Initial characterization to test for of basic motor and somatosensory function was performed as described by Rogers (24): righting reflex, wire hang, gait analysis, tail pinch and visual reach. Spatial memory testing was performed in the Morris water maze (25). Results for the male cKO mice were published in Oaks et al (16), and the females presented in Fig. 1 are littermates that were tested concurrently. A new behavioral cohort was generated for the PDE4D treatment. Behavioral analysis was performed via automated animal tracking using ANY-maze (Stoelting, Wood Dale IL).

Morris Water Maze-The Morris Water Maze (MWM) (16; 25) apparatus was a $120 \times 120 \mathrm{~cm}$ round metal tub (Stoelting, Wood Dale IL), where distinct visual cues were placed at the cardinal points. The surface of the water was made opaque by adding white non-toxic paint and the temperature was maintained at $24^{\circ} \mathrm{C}$. Each daily test consisted of four independent trials, one at each cardinal point around the tub, with the mouse being placed in the water facing the wall of the tub. Each trial lasted until the mouse found the platform or up to $60 \mathrm{~s}$, whichever occurred first. Each animal completed two daily tests with a visible platform to learn that a platform is available to escape from the water, then performed five tests with a platform hidden under the water surface to memorize the platform location based on the cardinal cues on the walls of the apparatus. After the hidden platform trials, a $60 \mathrm{~s}$ probe trial was performed by removing the platform and measuring the time spent swimming over the correct platform location. Finally, two reversal tests were completed after changing the location of the platform to test for cognitive flexibility and the 
ability to learn a new platform location. Mice were considered nonperformers and removed from the analysis when they refused to swim and floated in the water for $60 \mathrm{~s}$. Only animals that completed all trials were included.

\section{Signaling analysis via Western blot and ELISA}

Protein lysates were prepared from fresh, frozen mouse hippocampal or cortical tissue that was homogenized in a buffer containing Tris- $\mathrm{HCl}(50 \mathrm{mM}), \mathrm{NaCl}(100 \mathrm{mM})$, ethylenediaminetetraacetic acid $(5 \mathrm{mM})$, magnesium chloride $(2 \mathrm{mM})$, protease and phosphatase inhibitor mixture (Sigma-Aldrich, St. Louis MO) and extracted for $30 \mathrm{~min}$ with Triton X-100 (1\%) and sodium dodecyl sulfate (0.1\%). Lysates were cleared by centrifugation at $15,000 \mathrm{x} g$ for $20 \mathrm{~min}$ at $4^{\circ} \mathrm{C}$ then combined with one volume of Laemmli sample buffer (Bio-Rad, Hercules CA) containing 5\% beta-mercaptoethanol and denatured by heating at $95^{\circ} \mathrm{C}$ for $5 \mathrm{~min}$. Protein was separated by SDS-PAGE on 4-12\% Bis-Tris gels (Thermo Scientific, Waltham MA) and transferred to Immobilon-FL (Millipore, Billerica MA) polyvinylidine fluoride membranes. Immunoblots were probed with primary antibodies at optimized concentrations listed in Supplemental Table 1. Primary antibodies were then labeled with infra-red fluorophore-conjugated secondary antibodies (LI-COR Biosciences, Lincoln NE) and imaged on an Odyssey Imager (LI-COR Biosciences, Lincoln NE).

Cyclic AMP (cAMP) levels were measured using the Cyclic AMP Complete ELISA Kit (AbCam, ab133051, Cambridge, MA) following instructions from the manufacturer. Briefly, flash frozen hippocampal or cortical tissue was extracted in $0.1 \mathrm{M} \mathrm{HCl}$ and after $\mathrm{HCl}$ neutralization, mixed with anti-cAMP antibody and alkaline phosphatase for colorimetric detection in a Varioskan LUX multimode microplate reader (ThermoFisher Scientific, Waltham, MA).

\section{Protein immunoprecipitation}

Adult mouse forebrain tissue was homogenized by lysis buffer $(100 \mathrm{mM} \mathrm{NaCl}, 20 \mathrm{mM}$ Tris$\mathrm{Cl}$ (pH 7.4), $1 \mathrm{mM}$ EDTA, protease inhibitors 1:100) and centrifuged at 14,000×rpm for 10 min at $4^{\circ} \mathrm{C} .1 \mathrm{mg}$ of supernatant was incubated overnight at $4^{\circ} \mathrm{C}$ under rotary agitation with

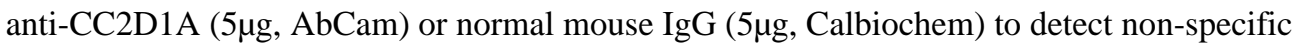
binding. Then, immunoprecipitated proteins were collected with $20 \mu \mathrm{L}$ of precleared unconjugated agarose beads (Vector Laboratories, Inc.). Finally, the beads were collected, washed 3 times with wash buffer $[150 \mathrm{mM} \mathrm{NaCl}, 20 \mathrm{mM}$ Tris-Cl (pH 7.4), protease inhibitors 1:100], resuspended with Laemmli sample buffer 2X (Bio-Rad, Hercules CA), boiled for $5 \mathrm{~min}$ at $95^{\circ} \mathrm{C}$, and separated by electrophoresis on 4-12\% Bis-Tris gels (Thermo Scientific, Waltham MA).

\section{Drug treatments}

Animals were treated with $3 \mu \mathrm{g} / \mathrm{kg}$ of GEBR-7b (Millipore, Burlington, MA) via intraperitoneal injection for 14 days prior to behavioral testing. Control animals were injected with saline only. Drug injections were continued every night during behavioral testing until the animals were sacrificed for tissue collection for Western blot analysis and ELISA, as described above. 


\section{RESULTS}

\section{Cc2d1a cKO mice show spatial memory deficits only in males}

Forebrain specific $C c 2 d 1 a \mathrm{cKO}$ mice were generated to circumvent early postnatal respiratory failure identified in global KO lines (20; 26-28) by removing Cc2d1a in the hippocampus and cortex via Cre-recombinase expressed under the CaMKIIa promoter as previously shown (16;21). Cc2d1a cKO animals are viable and fertile and show normal somatosensory and motor function in males and females (Suppl. Table 2). Control homozygous Cc2d1a-LoxP mice are listed as WT and Cc2d1a ${ }^{\text {CaMKIIa-cre mice are }}$ abbreviated as cKOs.

We had previously reported that $C c 2 d 1 a$ cKO males showed a deficit in spatial memory shown by a delay in memorizing the location of platform hidden under opaque water in the Morris Water Maze (MWM) (16). After an initial trial with a visible platform (VP) to learn that a platform is available to escape the water, each mouse is tested in multiple trials with a hidden platform (HP). The platform is then removed to test for permanence of the spatial memory (probe) and moved to a different location (reversal trial, RV) to assess cognitive flexibility $(25 ; 29)$. Cc2d1a cKO males had showed a defect in learning the HP location in days 2 and 3 of the 5-day hidden platform test, but had normal performance in the probe and RV test (Suppl. Table 3) (16). cKO females (N=21 WT, $17 \mathrm{cKO}$ ) showed equal performance to control animals in all phases of the test and demonstrated normal spatial memory (Fig.1AD). While sex has been shown not to affect performance in the MWM test in C57BL/6 mice (30), studies in rats showed that males and females can use different strategies to acquire the position of the hidden platform shifting from place-based strategies using visual cues, to motor-based search strategies such as scanning the length of tub (8). To confirm that females were not just using a different strategy to identify the location of the platform we compared learning strategies during the first three days of the HP test with our published male cKO cohort, using criteria outlined by Garthe et al (31). Thigmotaxis (swimming close to the sides of the tub), random path and scanning are considered motor strategies where the animal does not appear to use visual cues to locate the platform. Circling around the tub at the distance of the platform (chaining) or swimming toward the platform with (directed) or without errors (straight) indicate that the animal is using a place-based strategy. WT males and females showed no differences in using motor- (thigmotaxis, random search or scanning), and place-based strategies (chaining, directed search, or straight line) (Fig.1E). cKO males were slower in transitioning to place-based strategies in day 2 and 3 (Fig.1F-G). The primary factor driving the difference in performance in cKO males was the latency from drop location to the platform (Suppl. Table 3).

\section{CC2D1A controls signaling downstream of PDE4D in a male-specific manner}

Since spatial memory deficits have been linked to reduced CREB activation in the hippocampus (32) and CC2D1A is known to regulate CREB activation in hippocampal neurons by increasing PDE4D activity (20), we asked whether this pathway was altered in the adult hippocampus of $C c 2 d 1 a$-deficient mice (schematic in Fig.2A). We collected hippocampal tissue from 5 months old male and female WT and cKO mice. We tested for PDE4D, PKA, and CREB phosphorylation via Western blot by determining the ratio of 
phosphorylated to total protein in hippocampal protein lysates. We also separately measured tissue levels of cAMP using enzymelinked immunosorbent assay (ELISA) to confirm that PDE4D activity was altered. We found that, in fact, PDE4D phosphorylation was doubled in male $C c 2 d 1 a$-deficient hippocampi $(2.05 \pm 0.13$ of WT, $\mathrm{p}=0.0001 * * *, \mathrm{~N}=5 \mathrm{WT} ; 5 \mathrm{cKO}$, Fig. 2B). cAMP levels were almost halved (WT=51.83 $\pm 6.88 \mathrm{pmol} / \mathrm{mg}, \mathrm{N}=4$; $\mathrm{cKO}=27.74 \pm 1.92$ $\mathrm{pmol} / \mathrm{mg}, \mathrm{N}=4, \mathrm{p}=0.011^{*}$, Fig.2C). Finally, phosphorylation of Thr-197 in PKA $(0.48 \pm 0.13$ of WT, $\mathrm{p}=0.019 *, \mathrm{~N}=4 \mathrm{WT} ; 4$ cKO, Fig.2D) and Ser-133 in CREB $(0.70 \pm 0.09$ of WT, $\mathrm{p}=0.046 *, \mathrm{~N}=5 \mathrm{WT} ; 5 \mathrm{cKO}$, Fig.2E) were also decreased. These findings indicate that in the adult hippocampus lacking $C c 2 d 1 a$ PDE4D activity is increased leading to baseline reduction in cAMP levels and PKA and CREB phosphorylation which could underlie the cognitive deficits found in behavioral experiments.

When we tested the same biochemical changes in females, we found that there was no difference between WT and cKO animals (Fig.2F-H). Due to the high variability in CREB activity in female control hippocampi, we increased the number of animals tested to confirm that there was no difference and saw no change (CREB: cKO 0.93 \pm 0.11 of WT, $\mathrm{p}=0.69$, $\mathrm{N}=10 \mathrm{WT} ; 14$ cKO. Fig.1I). We tested for similar pathway alterations in cortical lysates where CC2D1A is also removed and found that PDE4D phosphorylation and cAMP levels were comparable to WT in cKO males and females (Suppl. Fig.1A-D). The amount of CC2D1A protein was measured in the cortex and hippocampus of WT animals and we found no differences in baseline CC2D1A levels between males and females (Suppl. Fig.1E). We also confirmed that CC2D1A binds to PDE4D in the male brain as previously shown (Fig. $3 \mathrm{~A})$ (19). We then wondered whether compensation could be present through $C c 2 d 1 b$, which is the only homolog of $C c 2 d 1 a$ in vertebrates, and has been shown to have a redundant and overlapping function $(22 ; 28)$. We tested the hippocampus in both $C c 2 d 1 b$ knock-out (KO) and $C c 2 d 1 a / C c 2 d 1 b$ double heterozygote (dHET) mice. We found that PDED4D phosphorylation was not altered in $C c 2 d 1 b$ KO animals (Fig.3B) and that it showed a trend towards being increased in the dHET animals (Fig.3C), suggesting that CC2D1A specifically controls PDE4D activity. We also confirmed that CC2D1B expression levels are not altered in $C c 2 d 1 a \mathrm{cKO}$ animals and that there is no difference in CC2D1A removal by CaMKII-acre mediated recombination in the male and female cortex and hippocampus (Suppl. Fig.1F-I). In summary, we found that loss of Cc2d1a causes male-specific activation of PDE4D, leading to reduced cAMP levels only in the hippocampus.

\section{Adult restoration of PDE4D activity rescues spatial memory deficits in Cc2d1a cKO males}

CREB activity is critical for creating and storing spatial memories (32-34) and its activation levels must be carefully controlled (35). Upstream of CREB, PDE4D is also a key regulator of cognitive function (36). We asked whether inhibiting PDE4D would reduce cAMP degradation to restore control levels of cAMP and CREB activity this rescuing spatial memory deficits in male cKOs (Fig.4A). PDE4 inhibitors have been studied in dementia and Alzheimer's Disease to slow cognitive decline by enhancing CREB function $(37 ; 38)$. However, the most commonly used drug, rolipram, targets multiple PDE4s and leads to severe side effects, including nausea and emesis which cause poor tolerability (37). To inhibit PDE4D in the cKO hippocampus we used a specific inhibitor GEBR-7b, which is selective for PDE4D and better tolerated (39). GEBR-7B treatment had proven effective in 
improving spatial memory in both WT mice and a mouse model of Alzheimer's Disease (39; 40). We chose a concentration of $3 \mu \mathrm{g} / \mathrm{kg}$ of GEBR-7b which would be able to restore normal cAMP levels without completely inhibiting PDE4D activity (Fig. 4). A cohort of adult ( 2 month-old) control and cKO male and female mice were treated for 14 days via intraperitoneal injections. Treatment was effective at restoring levels of cAMP as tested by ELISA (WT saline $=47.2 \pm 3.0 \mathrm{pmol} / \mathrm{mg}, \mathrm{N}=4, \mathrm{cKO}$ saline $=29.3 \pm 2.7 \mathrm{pmol} / \mathrm{mg}, \mathrm{N}=4, \mathrm{p}=0.014$ to WT; WT GEBR=46.4 $\pm 3.0 \mathrm{pmol} / \mathrm{mg}, \mathrm{N}=4, \mathrm{cKO}$ GEBR $=48.0 \pm 4.9 \mathrm{pmol} / \mathrm{mg}, \mathrm{N}=3$. Fig.4B) and levels of CREB phosphorylation as tested by Western blot (cKO saline $=0.47 \pm 0.08$ of WT saline, $\mathrm{p}=0.048 *$ * $\mathrm{N}=7 \mathrm{WT}, 6 \mathrm{cKO}$; WT GEBR=1.19 $\pm 0.26, \mathrm{~N}=6$; cKO GEBR $=0.82 \pm 0.07, N=7$. Fig. 4 C).

After treatment, the mice were tested in an abbreviated MWM protocol consisting of the visible, hidden, and probe tests, since neither male nor female cKO mice had shown a difference in the reversal test. In this cohort, cKO males again showed a delay in acquiring the position of the hidden platform when compared to controls in day 2 and the deficit was completely rescued by GEBR-7b treatment with an additional improvement for the $\mathrm{cKO}$ in day 1 (Fig. 5A-C. HP1 Fig. 5B: WT saline $=28.1 \pm 3.3 \mathrm{~s}, \mathrm{~N}=14$; $\mathrm{cKO}$ saline $=33.2 \pm 4.3 \mathrm{~s}, \mathrm{~N}=11$; WT GEBR $=28.2 \pm 3.8 \mathrm{~s}, \mathrm{~N}=14$; $\mathrm{cKO}$ GEBR $=18.3 \pm 2.0, \mathrm{~N}=14$, $\mathrm{p}$ values to $\mathrm{cKO}$ saline: WT saline $\mathrm{p}=0.4$ WT GEBR $\mathrm{p}=0.4$, cKO GEBR $\mathrm{p}<0.0001 * * *$;P2 Fig. $5 \mathrm{C}$ : WT saline $=9.6 \pm 1.3 \mathrm{~s} ; \mathrm{cKO}$ saline $=22.1 \pm 4.5 \mathrm{~s} ;$ WT GEBR $=13.2 \pm 2.0 \mathrm{~s} ; \mathrm{cKO}$ GEBR $=8.8 \pm 1.3 ; \mathrm{p}$ values to cKO saline: WT saline $\mathrm{p}=0.0005 * * *$, WT GEBR $\mathrm{p}=0.025 *$, cKO GEBR $\mathrm{p}=0.0002 * * *)$. Following spatial learning treated and untreated males performed equally in the probe trial (Fig.5D), and showed no differences in swim speeds showing that treatment did not change motor function (not shown). Females performed equally with and without GEBR-7b treatment (Fig.5E-H). Overall our results show that CC2D1A establishes malespecific CREB signaling through PDE4D that controls spatial memory acquisition.

\section{DISCUSSION}

In this study, we demonstrated that the ID/ASD gene CC2D1A controls male-specific CREB signaling in the hippocampus leading to male-specific spatial memory deficits in mice. We found that loss of CC2D1A in males increased hippocampal PDE4D phosphorylation leading to a reduction of cAMP levels and of CREB phosphorylation which can be rescued by PDE4D inhibition in cKO males. cKO female mice, however, do not show any of the signaling deficits observed in males and are not impaired in hidden platform acquisition in the MWM. Our findings identify a novel molecular mechanism for sex-specific signaling regulation controlled by a single gene mutated in ID and ASD.

Multiple hypotheses have been put forth to explain the male bias in neurodevelopmental disorders $(41 ; 42)$. Increased levels of steroid hormones, including cortisol and testosterone, and their precursors have been found elevated in the amniotic fluid of pregnancies that resulted in individuals with ASD (43), suggesting a role for sex hormones and other steroids in the etiology of the disorder in males. On the other hand, females require more severe rare genetic mutations and more familial risk factors than males to become affected $(44 ; 45)$, supporting the hypothesis that females are protected. A study by Grissom et al., in a mouse model of 16p11.2 deletion syndrome found male specific hyperactivity in MAPK3/ERK1 
kinase in the striatum correlating with male-specific striatal learning deficits (46), suggesting a link between sex-specific signaling and behavior. Our findings in Cc2d1acKO mice, together with those from the 16p11.2 hemideletion model (46), indicate that sex-specific disruptions in intracellular signaling may be involved in establishing male-specific behavioral phenotypes. While these findings do not yet define whether males are susceptible or females are protected, they show that in inbred genetic models recapitulating human mutations equally in males and females, males develop sex-specific signaling deficits. Defining how these signaling differences are established will not only provide insight into the mechanisms of sex bias in the pathogenesis of neurodevelopmental disorders, but will also likely identify sex-specific targets for therapy development.

We find that PDE4D inhibition can rescue cognitive deficits in a model of neurodevelopmental disorders. PDE4 proteins have been under investigation as a target for the improvement of cognitive function in neurodegenerative disorders for some time, since CREB activity is disrupted in multiple models of Alzheimer's disease (AD) $(37 ; 38 ; 47)$. It will be important to determine whether PDE4D function is disrupted in other models of neurodevelopmental disease, and whether PDE4D inhibition could be beneficial in ID. It should also be noted that $\mathrm{AD}$ and dementia show a sex-bias which is opposite than the bias observed in ID and ASD, with females comprising two thirds of individuals affected by AD (48). Studies in primary hippocampal neurons showed that CREB is primarily controlled by estrogen receptor activation in conjunction with metabotropic glutamate receptors in female neurons, and not in males (10). Sex differences in the regulation of PDE4D and CREB signaling at different ends of the human life-span may need to considered in developing therapies as similar mechanisms may be involved in cognitive development and dementia.

To date, CC2D1A mutations have been identified in 34 individuals ( 21 males and 13 females) with fully penetrant developmental delay and ID in males and females, and partially penetrant reports of ASD, ADHD, aggressive behavior, and seizures, primarily in males (12-15). Since most of the individuals with CC2D1A mutations reside in the Middle East and are not currently accessible to follow-up, we do not know whether there are significant differences in the behavioral presentation and severity between males and females. Intriguing sex-specific differences in CC2D1A expression were found in Major Depressive Disorder (MDD). Conditional $C c 2 d 1$ a removal in serotoninergic neurons in the dorsal raphe demonstrated that $\mathrm{CC} 2 \mathrm{D} 1 \mathrm{~A}$ also has a role in regulating serotonin receptor function and depression-like behaviors (49). CC2D1A levels were found reduced in the prefrontal cortex (50) and increased in the cingulate cortex (51) in males, but not females affected by MDD, suggesting that male-specific impairment in CC2D1A function in humans could be present. CC2D1A expression was elevated in the blood of individuals with ASD (52), but the number of females in the sample was too small to explore sexspecificity of this change and more extensive follow-up will be necessary. PDE4D expression is also regulated by both serotonin and norepinephrine reuptake inhibitors (53; 54), suggesting a shared role for CC2D1A and PDE4D in cognition and depression. CC2D1A has also been studied in regulation of subcellular and endosomal regulation of signaling proteins $(20 ; 55 ; 56)$ and future investigations should focus on whether this function is regulated by sex hormones and/or involved in sex hormone function in conjunction with neuronal activity and memory 
formation. Defining how these mechanisms control cognitive and affective function throughout life may provide insight into sex bias in disease susceptibility.

\section{Supplementary Material}

Refer to Web version on PubMed Central for supplementary material.

\section{ACKNOWLEDGEMENTS}

The authors are grateful to the Animal Research Facility at GWU SMHS, Judy Liu, Thomas Maynard, Maria Chahrour, Ottavio Arancio, and Emanuela Santini for helpful discussion on experimental design. This work was supported by start-up funds from the George Washington University, NIH grants R00HD067379 and R01NS105000, and Pilot Grants from the Intellectual and Developmental Disabilities Research Center (IDDRC) at Children's Research Institute (P30HD040677) and from the Clinical and Translational Studies Institute at Children's National (UL1TR001876), all to M.C.M. The Cc2d1a and Cc2d1b KO mouse strains used for this research project was generated by the trans-NIH Knock-Out Mouse Project (KOMP) and obtained from the KOMP Repository (www.komp.org). NIH grants to Velocigene at Regeneron Inc (U01HG004085) and the CSD Consortium (U01HG004080) funded the generation of gene-targeted ES cells for 8500 genes in the KOMP Program and archived and distributed by the KOMP Repository at UC Davis and CHORI (U42RR024244). For more information or to obtain KOMP products go to www.komp.org or service@ komp.org.

\section{REFERENCES}

1. Baio J, Wiggins L, Christensen DL, Maenner MJ, Daniels J, Warren Z, et al. (2018): Prevalence of Autism Spectrum Disorder Among Children Aged 8 Years - Autism and Developmental Disabilities Monitoring Network, 11 Sites, United States, 2014. MMWR Surveill Summ. 67: 1-23.

2. Fombonne E (2005): Epidemiology of autistic disorder and other pervasive developmental disorders. J Clin Psychiatry. 66 Suppl 10: 3-8.

3. McCarthy MM (2016): Sex differences in the developing brain as a source of inherent risk. Dialogues Clin Neurosci. 18: 361-372. [PubMed: 28179808]

4. Tunç B, Solmaz B, Parker D, Satterthwaite TD, Elliott MA, Calkins ME, et al. (2016): Establishing a link between sex-related differences in the structural connectome and behaviour. Philos Trans $\mathrm{R}$ Soc Lond, B, Biol Sci. 371: 20150111. [PubMed: 26833832]

5. Björnsdotter M, Wang N, Pelphrey K, Kaiser MD (2016): Evaluation of Quantified Social Perception Circuit Activity as a Neurobiological Marker of Autism Spectrum Disorder. JAMA Psychiatry. 73: 614-621. [PubMed: 27096285]

6. Forgie ML, Kolb B (1998): Sex differences in the effects of frontal cortex injury: role of differential hormonal experience in early development. Behav Neurosci. 112: 141-153. [PubMed: 9517822]

7. Roof RL, Zhang Q, Glasier MM, Stein DG (1993): Gender-specific impairment on Morris water maze task after entorhinal cortex lesion. Behav Brain Res. 57: 47-51. [PubMed: 8292254]

8. Schmidt B, Jacobson TK, Markus E (2009): Hippocampal and striatal dependent navigation: sex differences are limited to acquisition. Horm Behav. 56: 199-205. [PubMed: 19406124]

9. Keiser AA, Turnbull LM, Darian MA, Feldman DE, Song I, Tronson NC (2017): Sex Differences in Context Fear Generalization and Recruitment of Hippocampus and Amygdala during Retrieval. Neuropsychopharmacology. 42: 397-407. [PubMed: 27577601]

10. Boulware MI, Weick JP, Becklund BR, Kuo SP, Groth RD, Mermelstein PG (2005): Estradiol activates group I and II metabotropic glutamate receptor signaling, leading to opposing influences on cAMP response element-binding protein. Journal of Neuroscience. 25: 5066-5078. [PubMed: 15901789]

11. Frick KM, Kim J, Tuscher JJ, Fortress AM (2015): Sex steroid hormones matter for learning and memory: estrogenic regulation of hippocampal function in male and female rodents. Learn Mem. 22: 472-493. [PubMed: 26286657]

12. Basel-Vanagaite L, Attia R, Yahav M, Ferland RJ, Anteki L, Walsh CA, et al. (2006): The CC2D1A, a member of a new gene family with C2 domains, is involved in autosomal recessive non-syndromic mental retardation. J Med Genet. 43: 203-210. [PubMed: 16033914] 
13. Manzini MC, Xiong L, Shaheen R, Tambunan DE, Di Costanzo S, Mitisalis V, et al. (2014): CC2D1A Regulates Human Intellectual and Social Function as well as NF- $\kappa$ B Signaling Homeostasis. Cell Rep. 8: 647-655. [PubMed: 25066123]

14. Reuter MS, Tawamie H, Buchert R, Hosny Gebril O, Froukh T, Thiel C, et al. (2017): Diagnostic Yield and Novel Candidate Genes by Exome Sequencing in 152 Consanguineous Families With Neurodevelopmental Disorders. JAMA Psychiatry. 74: 293-299. [PubMed: 28097321]

15. Loviglio MN, Beck CR, White JJ, Leleu M, Harel T, Guex N, et al. (2016): Identification of a RAI1-associated disease network through integration of exome sequencing, transcriptomics, and 3D genomics. Genome Med. 8: 105. [PubMed: 27799067]

16. Oaks AW, Zamarbide M, Tambunan DE, Santini E, Di Costanzo S, Pond HL, et al. (2017): Cc2d1a Loss of Function Disrupts Functional and Morphological Development in Forebrain Neurons Leading to Cognitive and Social Deficits. Cereb Cortex. 27: 1670-1685. [PubMed: 26826102]

17. Matsuda A, Suzuki Y, Honda G, Muramatsu S, Matsuzaki O, Nagano Y, et al. (2003): Largescale identification and characterization of human genes that activate NF-kappaB and MAPK signaling pathways. Oncogene. 22: 3307-3318. [PubMed: 12761501]

18. Ou X-M, Lemonde S, Jafar-Nejad H, Bown CD, Goto A, Rogaeva A, Albert PR (2003): Freud-1: A neuronal calcium-regulated repressor of the 5-HT1A receptor gene. Journal of Neuroscience. 23: 7415-7425. [PubMed: 12917378]

19. Al-Tawashi A, Jung SY, Liu D, Su B, Qin J (2012): Protein implicated in nonsyndromic mental retardation regulates protein kinase A (PKA) activity. Journal of Biological Chemistry. 287: 14644-14658. [PubMed: 22375002]

20. Al-Tawashi A, Gehring C (2013): Phosphodiesterase activity is regulated by CC2D1A that is implicated in non-syndromic intellectual disability. Cell Commun Signal. 11: 47. [PubMed: 23826796]

21. Tsien JZ, Chen DF, Gerber D, Tom C, Mercer EH, Anderson DJ, et al. (1996): Subregion- and cell type-restricted gene knockout in mouse brain. Cell. 87: 1317-1326. [PubMed: 8980237]

22. Zamarbide M, Oaks AW, Pond HL, Adelman JS, Manzini MC (2018): Loss of the Intellectual Disability and Autism GeneCc2d1aand Its HomologCc2d1bDifferentially Affect Spatial Memory, Anxiety, and Hyperactivity. Front Genet. 9: 65. [PubMed: 29552027]

23. Choi C-I, Yoon S-P, Choi J-M, Kim S-S, Lee Y-D, Birnbaumer L, Suh-Kim H (2014): Simultaneous deletion of floxed genes mediated by CaMKIIa-Cre in the brain and in male germ cells: application to conditional and conventional disruption of Goa. Exp Mol Med. 46: e93. [PubMed: 24787734]

24. Rogers DC, Fisher EM, Brown SD, Peters J, Hunter AJ, Martin JE (1997): Behavioral and functional analysis of mouse phenotype: SHIRPA, a proposed protocol for comprehensive phenotype assessment. Mamm Genome. 8: 711-713. [PubMed: 9321461]

25. Vorhees CV, Williams MT (2006): Morris water maze: procedures for assessing spatial and related forms of learning and memory. Nat Protoc. 1: 848-858. [PubMed: 17406317]

26. Zhao M, Raingo J, Chen ZJ, Kavalali ET (2011): Cc2d1a, a C2 domain containing protein linked to nonsyndromic mental retardation, controls functional maturation of central synapses. Journal of Neurophysiology. 105: 1506-1515. [PubMed: 21273312]

27. Chen K-R, Chang C-H, Huang C-Y, Lin C-Y, Lin W-Y, Lo Y-C, et al. (2012): TBK1-associated protein in endolysosomes (TAPE)/CC2D1A is a key regulator linking RIG-I-like receptors to antiviral immunity. Journal of Biological Chemistry. 287: 32216-32221. [PubMed: 22833682]

28. Drusenheimer N, Migdal B, Jäckel S, Tveriakhina L, Scheider K, Schulz K, et al. (2015): The Mammalian Orthologs of Drosophila Lgd, CC2D1A and CC2D1B, Function in the Endocytic Pathway, but Their Individual Loss of Function Does Not Affect Notch Signalling. (MartinezArias A, editor) PLoS Genet. 11: e1005749. [PubMed: 26720614]

29. Morris R (1984): Developments of a water-maze procedure for studying spatial learning in the rat. J Neurosci Methods. 11: 47-60. [PubMed: 6471907]

30. Baldan Ramsey LC, Pittenger C (2010): Cued and spatial learning in the water maze: equivalent learning in male and female mice. Neurosci Lett. 483: 148-151. [PubMed: 20691760] 
31. Garthe A, Behr J, Kempermann G (2009): Adult-generated hippocampal neurons allow the flexible use of spatially precise learning strategies. (Earley RL, editor) PLoS ONE. 4: e5464. [PubMed: 19421325]

32. Bourtchuladze R, Frenguelli B, Blendy J, Cioffi D, Schütz G, Silva AJ (1994): Deficient longterm memory in mice with a targeted mutation of the cAMP-responsive element-binding protein. Cell. 79: 59-68. [PubMed: 7923378]

33. Barco A, Alarcon JM, Kandel ER (2002): Expression of constitutively active CREB protein facilitates the late phase of long-term potentiation by enhancing synaptic capture. Cell. 108: 689703. [PubMed: 11893339]

34. Kida S, Josselyn SA, Peña de Ortiz S, Kogan JH, Chevere I, Masushige S, Silva AJ (2002): CREB required for the stability of new and reactivated fear memories. Nat Neurosci. 5: 348-355. [PubMed: 11889468]

35. Viosca J, Malleret G, Bourtchouladze R, Benito E, Vronskava S, Kandel ER, Barco A (2009): Chronic enhancement of CREB activity in the hippocampus interferes with the retrieval of spatial information. Learn Mem. 16: 198-209. [PubMed: 19237642]

36. Ricciarelli R, Fedele E (2015): Phosphodiesterase 4D: an enzyme to remember. Br J Pharmacol. 172: 4785-4789. [PubMed: 26211680]

37. Teich AF, Nicholls RE, Puzzo D, Fiorito J, Purgatorio R, Fa M, Arancio O (2015): Synaptic therapy in Alzheimer's disease: a CREB-centric approach. Neurotherapeutics. 12: 29-41. [PubMed: 25575647]

38. Gurney ME, D’ Amato EC, Burgin AB (2015): Phosphodiesterase-4 (PDE4) molecular pharmacology and Alzheimer's disease. Neurotherapeutics. 12: 49-56. [PubMed: 25371167]

39. Bruno O, Fedele E, Prickaerts J, Parker LA, Canepa E, Brullo C, et al. (2011): GEBR-7b, a novel PDE4D selective inhibitor that improves memory in rodents at non-emetic doses. Br J Pharmacol. 164: 2054-2063. [PubMed: 21649644]

40. Sierksma ASR, van den Hove DLA, Pfau F, Philippens M, Bruno O, Fedele E, et al. (2014): Improvement of spatial memory function in APPswe/PS1dE9 mice after chronic inhibition of phosphodiesterase type 4D. Neuropharmacology. 77: 120-130. [PubMed: 24067928]

41. Baron-Cohen S, Lombardo MV, Auyeung B, Ashwin E, Chakrabarti B, Knickmeyer R (2011): Why are autism spectrum conditions more prevalent in males? PLoS Biol. 9: e1001081. [PubMed: 21695109]

42. Werling DM, Geschwind DH (2013): Understanding sex bias in autism spectrum disorder. Proc Natl Acad Sci USA. 110: 4868-4869. [PubMed: 23476067]

43. Baron-Cohen S, Auyeung B, Nørgaard-Pedersen B, Hougaard DM, Abdallah MW, Melgaard L, et al. (2015): Elevated fetal steroidogenic activity in autism. Mol Psychiatry. 20: 369-376. [PubMed: 24888361]

44. Gilman SR, Iossifov I, Levy D, Ronemus M, Wigler M, Vitkup D (2011): Rare de novo variants associated with autism implicate a large functional network of genes involved in formation and function of synapses. Neuron. 70: 898-907. [PubMed: 21658583]

45. Robinson EB, Lichtenstein P, Anckarsäter H, Happé F, Ronald A (2013): Examining and interpreting the female protective effect against autistic behavior. Proc Natl Acad Sci USA. 110: 5258-5262. [PubMed: 23431162]

46. Grissom NM, McKee SE, Schoch H, Bowman N, Havekes R, O’Brien WT, et al. (2017): Malespecific deficits in natural reward learning in a mouse model of neurodevelopmental disorders. Mol Psychiatry. 177: 785.

47. Vitolo OV, Sant'Angelo A, Costanzo V, Battaglia F, Arancio O, Shelanski M (2002): Amyloid beta -peptide inhibition of the PKA/CREB pathway and long-term potentiation: reversibility by drugs that enhance cAMP signaling. Proc Natl Acad Sci USA. 99: 13217-13221. [PubMed: 12244210]

48. Mielke MM, Vemuri P, Rocca WA (2014): Clinical epidemiology of Alzheimer's disease: assessing sex and gender differences. Clin Epidemiol. 6: 37-48. [PubMed: 24470773]

49. Vahid-Ansari F, Daigle M, Manzini MC, Tanaka KF, Hen R, Geddes SD, et al. (2017): Abrogated Freud-1/CC2D1A repression of 5-HT1A autoreceptors induces fluoxetine-resistant anxiety/ depression-like behavior. Journal of Neuroscience. 37: 11967-11978. [PubMed: 29101244] 
50. Szewczyk B, Albert PR, Rogaeva A, Fitzgibbon H, May WL, Rajkowska G, et al. (2010): Decreased expression of Freud-1/CC2D1A, a transcriptional repressor of the 5-HT1A receptor, in the prefrontal cortex of subjects with major depression. Int J Neuropsychopharmacol. 13: 10891101. [PubMed: 20392296]

51. Labonté B, Engmann O, Purushothaman I, Menard C, Wang J, Tan C, et al. (2017): Sex-specific transcriptional signatures in human depression. Nat Med. 31: 212.

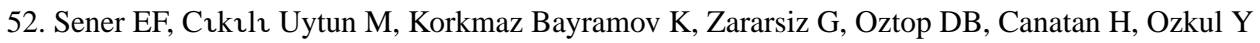
(2016): The roles of CC2D1A and HTR1A gene expressions in autism spectrum disorders. Metab Brain Dis. 31: 613-619. [PubMed: 26782176]

53. Zhang H-T, Huang Y, Jin S-L, Frith SA, Suvarna N, Conti M, O’Donnell JM (2002): Antidepressant-like profile and reduced sensitivity to rolipram in mice deficient in the PDE4D phosphodiesterase enzyme. Neuropsychopharmacology. 27: 587-595. [PubMed: 12377395]

54. Dlaboga D, Hajjhussein H, O’Donnell JM (2006): Regulation of phosphodiesterase-4 (PDE4) expression in mouse brain by repeated antidepressant treatment: comparison with rolipram. Brain Res. 1096: 104-112. [PubMed: 16730340]

55. Jaekel R, Klein T (2006): The Drosophila Notch inhibitor and tumor suppressor gene lethal (2) giant discs encodes a conserved regulator of endosomal trafficking. Developmental Cell. 11: 655669. [PubMed: 17084358]

56. Gallagher CM, Knoblich JA (2006): The conserved c2 domain protein lethal (2) giant discs regulates protein trafficking in Drosophila. Developmental Cell. 11: 641-653. [PubMed: 17084357] 

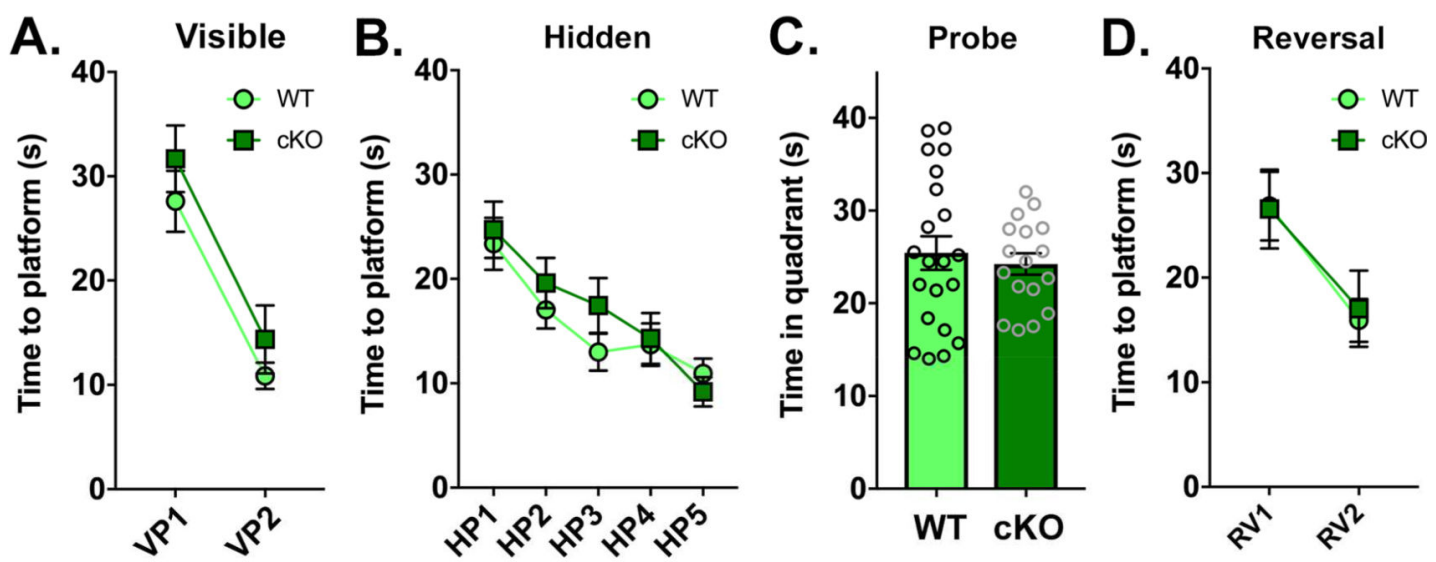

\section{E. Thigmotaxis

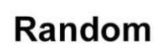 \\ Scanning}

\section{Chaining}

Directed

Straight

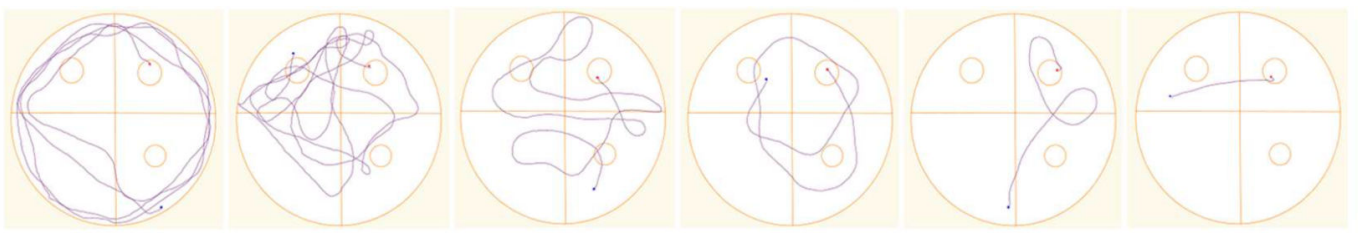

$\mathbf{F}$

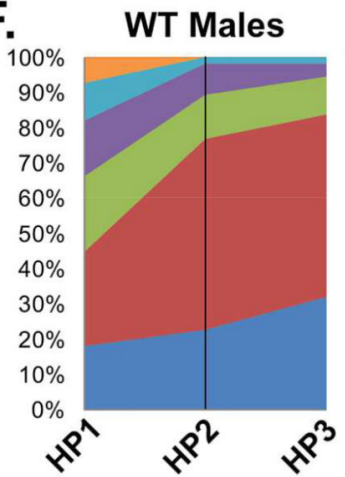

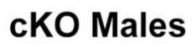

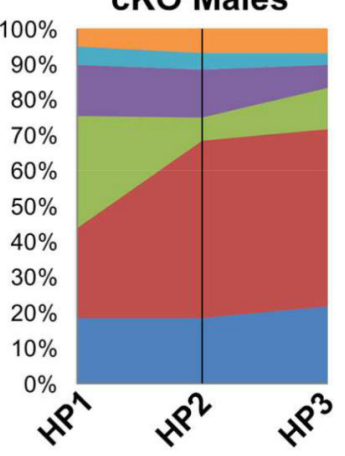

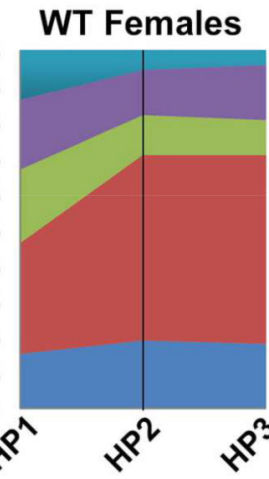

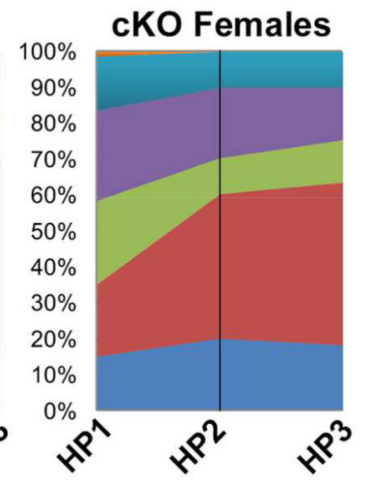

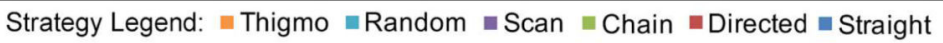

Figure 1. $C c 2 d 1 a$ cKO females do not show a deficit in spatial memory.

A-D. Hippocampusdependent spatial memory was assessed in cKO females via the Morris Water Maze test. Spatial learning was measured as latency to reach the platform in two different stages, visible platform (VP, A.) and hidden platform (HP, B.). Spatial memory retention was measured in the Probe trial (C.) and flexibility in the reversal (RV, D.) trial. Differently from cKO males which show a delay in the HP trial in Oaks et al. (16), cKO females showed no deficit (Suppl. Table 3). E. Six different learning strategies were assayed using criteria from Garthe et al (31). F. Average use of different strategies is broken down for males and females during the first three HP trials showing that $\mathrm{cKO}$ males maintain motor strategies such as thigmotaxis, scanning and random search longer than females. 
A.

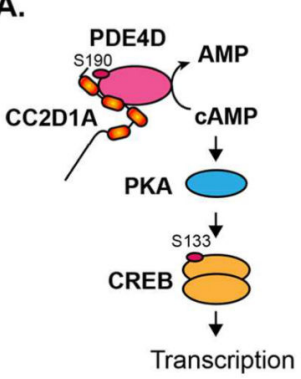

B.

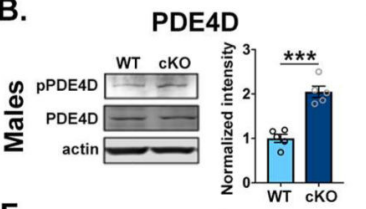

$\mathbf{F}$

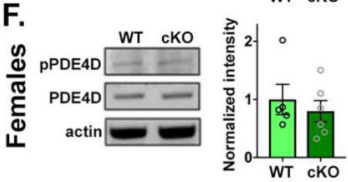

c.

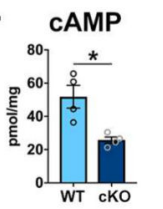

G.

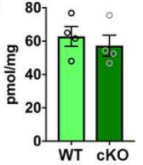

D.

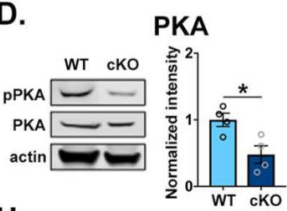

H.

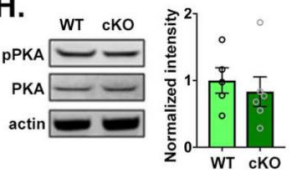

E.

CREB

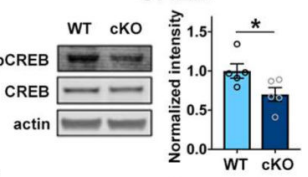

I.

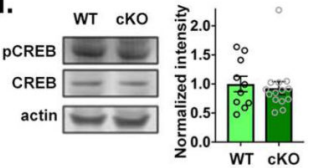

Figure 2. Loss of Cc2d1a causes male-specific disruption of PDE4D and CREB activity in the hippocampus.

A. Schematic of CC2D1A as a signaling scaffold for PDE4D and PKA upstream of CREB. B-E. Conditional removal of $C c 2 d 1 a$ in $\mathrm{cKO}$ male mice causes increased phosphorylation and activity of PDE4D (B.), leading to reduced levels of cAMP (C.), and decreased phosphorylation of both PKA (D.) and CREB (E.) in hippocampal lysates. F-I. No changes in PDE4D (F.), cAMP (G.), PKA (H.) or CREB (I.) are found in the female hippocampus. Results expressed as mean \pm SEM. Data averages and statistical information are reported in the Results. Two-tailed t-test with equal variance * $\mathrm{p}<0.05, * * \mathrm{p}<0.01, * * * \mathrm{p}<0.001$ 
A. input IP+ IP.

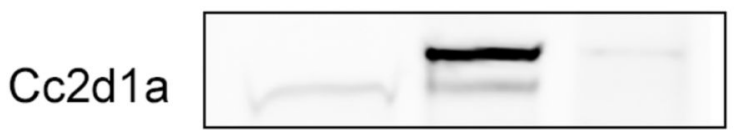

PDE4D

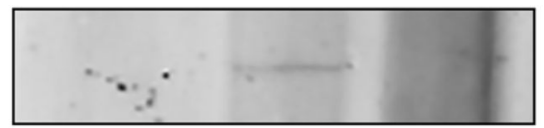

B.

Cc2d1b KO

WT

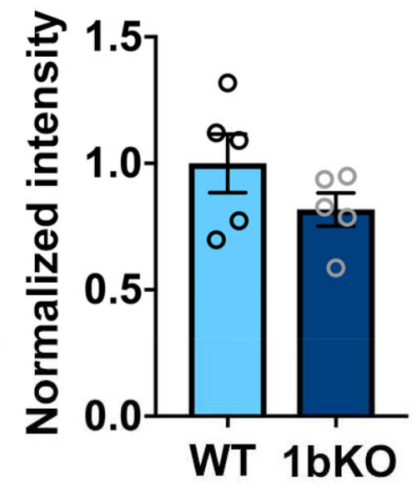

pPDE4D

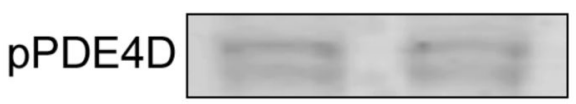

PDE4D

actin

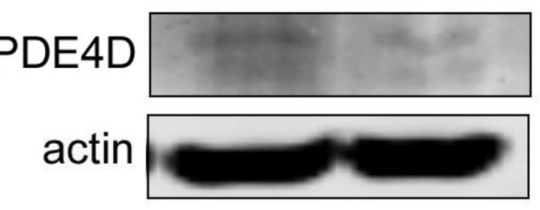

KO

C. Cc2d1a/1b dHET

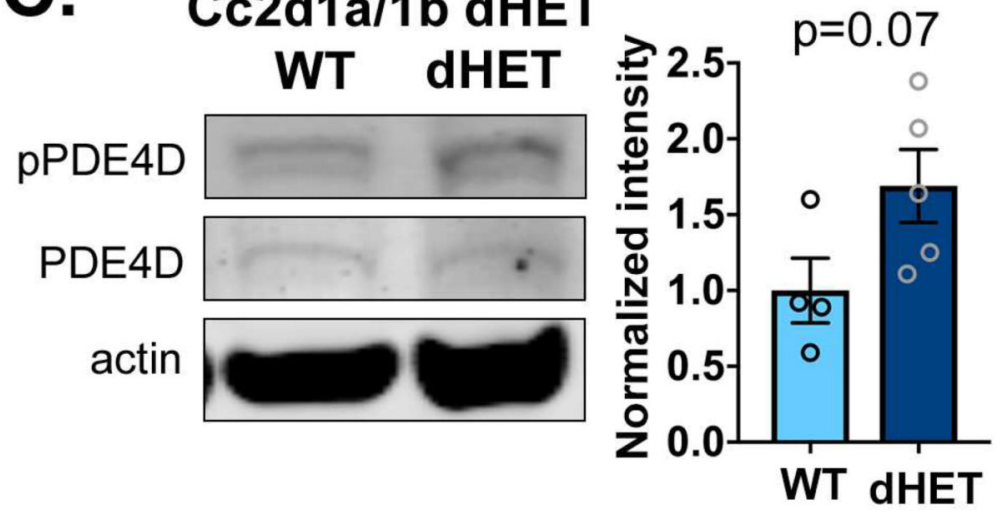

Figure 3. Regulation of PDE4D phosphorylation is specific to CC2D1A.

A. PDE4D immunoprecipitates with CC2D1A in brain lysates (IP+). B-C. PDE4D phosphorylation is not changed in the $\mathrm{Cc} 2 \mathrm{~d} 1 \mathrm{~b} \mathrm{KO}$ hippocampus (B.) but shows a trend towards being increased when expression of both $\mathrm{Cc} 2 \mathrm{~d} 1 \mathrm{a}$ and $\mathrm{Cc} 2 \mathrm{~d} 1 \mathrm{~b}$ is halved in the Cc2d1a/1b dHET animals (C.). 


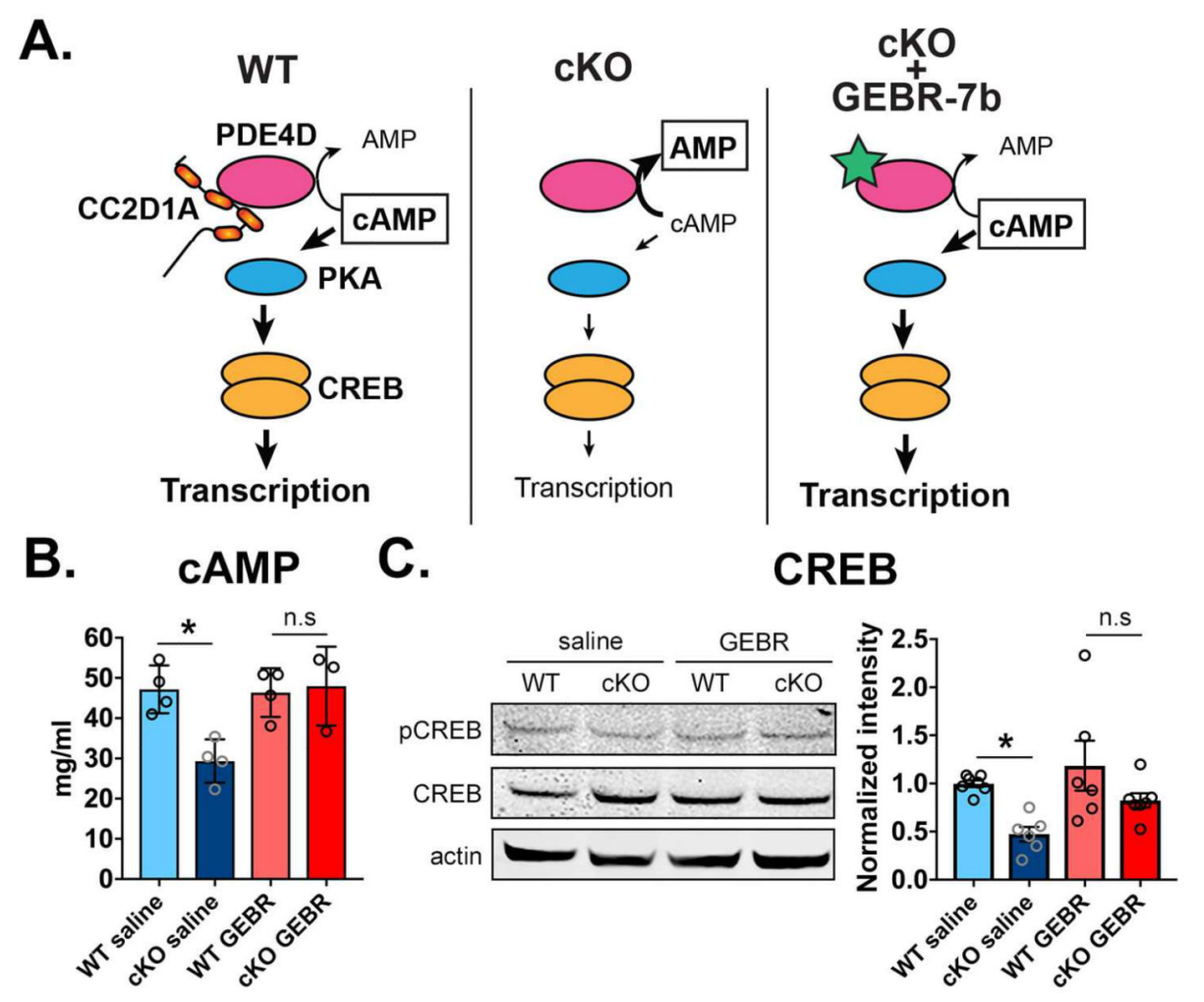

Figure 4. GEBR-7b treatment restores cAMP levels and CREB phosphorylation in $C c 2 d 1 a$ cKO males.

A. Schematic of expected action of GEBR-7b (green star). When CC2D1A is absent hyperactivity of PDE4D in cKO males increases cAMP degradation (center panel). GEBR-7b is expected to restore cAMP levels and CREB-mediated transcription (right panel). B. 14-day daily treatment with $3 \mu \mathrm{g} / \mathrm{kg}$ of GEBR-7b (GEBR) restored cAMP to WT levels in the hippocampus of cKO males when compared to animals treated with saline. $\mathbf{C}$. CREB phosphorylation is also increased in GEBR-7b treated cKO males. One-way ANOVA with Tukey's multiple comparison test, * $\mathrm{p}<0.05$. 
A. Visible

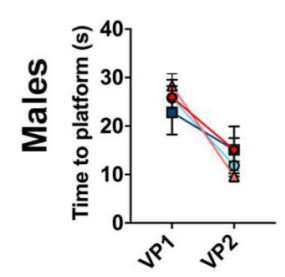

E.

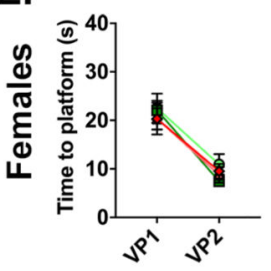

Hidden
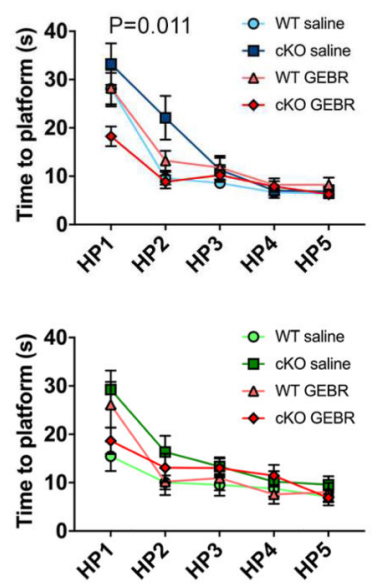

B.
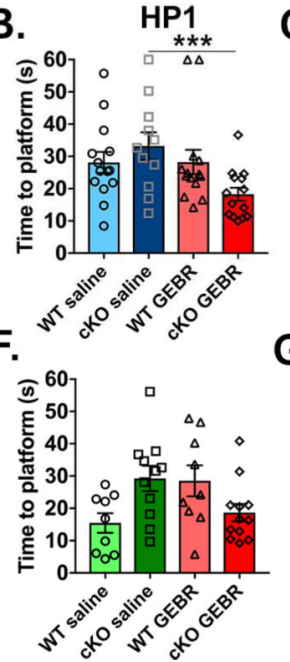

C.

G.

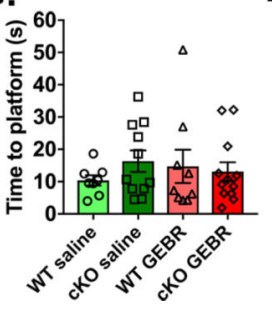

D.

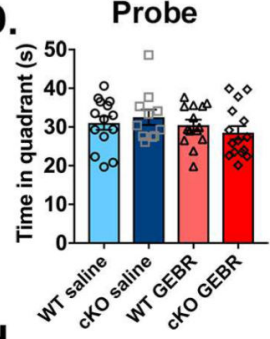

H.

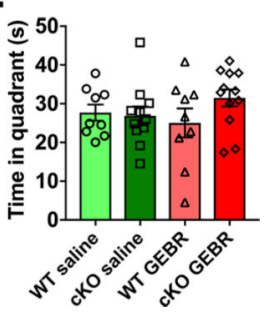

Figure 5. GEBR-7b treatment rescues spatial memory deficit in Cc2d1a cKO males and has no effect on females.

A. The learning delay in the hidden platform (HP) trial of the MWM found in male Cc2d1a cKO animals is corrected by $3 \mu \mathrm{g} / \mathrm{kg}$ GEBR-7b treatment for 14 days before testing. Two way ANOVA effect of treatment in hidden platform test $\mathrm{p}=0.011$. B-C. Significant improvement following GEBR-7b treatment are found in both HP1 and HP2 testing days via one way ANOVA for individual days. $* * * p<0.001$ D. No significant change is found in the Probe test. E-H. GEBR-7b treatment has no effect on female mice. 